\title{
Tetralogy of Fallot, Pulmonary Valve Stenosis, Ventricular Septal Defect, and Hypertrophic Cardiomyopathy in WKY/NCrj Rats
}

\author{
TOSHIRO KURIBAYASHI, KAZUTOSHI SHIMOO, TAKASHI NAKAMURA, \\ HIROFUMI TANIWAKI, KENJI HAMAOKA, MASAO NAKAGAWA, YASUHIKO IBATA, \\ TOMOHIKO KOMEDA, AND AKINOBU NAGAOKA \\ Second Department of Anatomy [T.Ku., Y.I.], Second Department of Medicine [K.S., T.N., M.N.], Third \\ Department of Medicine [H.T.], and Children's Research Hospital [K.H.], Kyoto Prefectural University of \\ Medicine, Kyoto, and Central Research Division [T.Ko., A.N.J Takeda Chemical Industries Ltd., Osaka, Japan
}

\begin{abstract}
We examined anatomically the hearts of 198 WKY/NCrj rats of 20 litters. There were 51 rats with moderate to severe thickening of the pulmonary valve and 19 rats with a ventricular septal defect; the two lesions occurred together in 16 rats, in 15 of which there were overriding of the aorta, stenosis of the pulmonary outflow tract, and hypertrophy of the right ventricle, fulfilling the criteria for tetralogy of Fallot in man. The papillary muscle of the conus was absent in 65 rats. The heart was abnormally heavy in 18 . We analyzed the relationship between cardiac hypertrophy and valvular lesions and septal defects in these rats plus 27 selected WKY rats with abnormally heavy hearts. Of the 151 rats with neither severe valvular lesions nor septal defects, six rats had abnormally heavy hearts and 67 rats had disproportionate ventricular septal thickening. This situation in the rats is similar to hypertrophic cardiomyopathy. The occurrence of these abnormalities, isolated or in association, in rats of an established inbred strain strongly suggests that they are etiologically or genetically linked, and that the rats should be a useful animal model for these diseases in man. (Pediatr Res 27 483-487, 1990)
\end{abstract}

\section{Abbreviations}

P-valve, pulmonary valve

VSD, ventricular septal defect

POT, pulmonary outflow tract

TOF, tetralogy of Fallot

DST, disproportionate ventricular septal thickening

RVH, right ventricular hypertrophy

RVFW, right ventricular free wall

LVFW, left ventricular free wall

IVS, ventricular septum

Stenosis of the P-valve and/or POT, VSD, overriding of the aorta, and RVH are well known to occur in humans with their full association being termed TOF $(1-6)$. These lesions have been reported in animals also, i.e. TOF in Keeshond dogs (7-9), Pvalve stenosis in beagle dogs (10), and VSD in Long-Evans rats

Received November 7, 1989; accepted December 11, 1989.

Correspondence and reprint requests: Toshiro Kuribayashi, M.D., Second Department of Anatomy, Kyoto Prefectual University of Medicine, KawaramachiHirokoji Kamigyo-Ku Kyoto 602, Japan.

Supported in part by Grant No. 62580034 from the Ministry of Education, Science, and Culture of Japan.
(11); they have been considered animal models for the human congenital heart diseases.

Our report describes these cardiac anomalies that occur spontaneously in rats of an inbred strain. We have already reported that various myocardial abnormalities similar to human HCM occur in the majority of these animals, e.g. cardiac hypertrophy, DST, muscle fiber disarrangement, myocardial fibrosis, coronary arterial wall thickening, and/or narrow left ventricular cavity $(12,13)$. These anomalous lesions, similar to TOF, were overlooked in our previous macroscopic observations.

\section{MATERIALS AND METHODS}

Animals. We used $225 \mathrm{WKY} / \mathrm{NCrj}$ rats: 198 rats of 20 litters obtained by random breeding and 27 rats with marked cardiac hypertrophy selected from 293 rats obtained in the process of selective breeding for hypertrophy on the basis of electrocardiographic findings (13). They were 8-40 wk old and weighed 130 $420 \mathrm{~g}$. They had been raised in an air-conditioned room free from specific pathogens. The results were compared with those of 30 wt-matched adult Wistar rats with normal hearts from Shimizu Jikken Zairyo Inc. (Kyoto, Japan) $(12,13)$.

Methods. Under urethane anesthesia, the heart was arrested at end-diastole by an injection of saturated EDTA solution (0.1$2.0 \mathrm{~mL}$ ) into the right atrium. The right atrium was incised to allow drainage of the perfusate, and the coronary arteries were immediately perfused with $10 \%$ formalin through the cannulated carotid artery under a perfusion pressure maintained at $80 \mathrm{~mm}$ $\mathrm{Hg}$. The heart was weighed without the atria and then manually cut transversely midway between the apex and the aortic root in order to measure the thickness of the LVFW and IVS and the transverse wall area of the RVFW in the same way as in the previous studies $(12,13)$.

After dissection of the great vessels, we observed the semilunar valves from the distal side and the atrioventricular valves from the ventricular side under a dissecting microscope. The thickening of the $P$-valve was classified as: grade 0 , no thickening; grade 1 , mild thickening of the cusps limited to their tips, which were obviously thicker than normally existing nodules; grade 2 , obvious thickening of the cusp edges; grade 3, severe thickening over a wide area of the cusps with distortion and impaired apposition of the cusps with or without fusion of the commissures (Fig. 1). Several P-valves with grade 3 thickening were examined histologically after Masson-Goldner's trichrome staining.

The RVFW was then dissected so that the IVS, tricuspid valves, papillary muscles, and POT could be examined (Fig. 2). The major and minor diameters of the POT and VSD were measured from the right ventricle with an eye-piece micrometer to calculate 
areas. Membranous tissues that were very often present in VSD were excluded from the measurement.

Data analysis. Males and females were analyzed together because there were no significant differences between sexes. The incidence of each lesion was determined in the 198 rats of 20 litters. The relationship between various lesions was analyzed in 225 rats, the 198 plus 27 selected rats with heavy hearts which were usually full of lesions. The heart wt was corrected by body wt raised to the power of 0.9 ; this correction was done to normalize the heart wt of rats with a wide range of body wt. The mean values between the WKY and Wistar rats were not compared due to $F$ ratios of more than $5 \%$ in a vast majority of the pairs. An individual value in a WKY rat was judged to be abnormal when it was outside the mean \pm 2 SD of the value for the Wistar rats. The mean values in the multiple groups were compared by Duncan's multiple comparison test. The percentage of WKY rats with an abnormal value or a lesion was compared by the $\chi^{2}$ test. Correlation coefficients were expressed as a $95 \%$ confidence interval.

\section{RESULTS}

Valves. There were $\mathrm{P}$-valves with grade 1 and grade 2-3 thickening in 33 and $26 \%$, respectively, of the nonselected 198 WKY rats (Table 1). Dome-shaped valves were not found in any of the 225 rats. In 66 of the 68 rats with grade 2-3 P-valve thickening, commissural fusion of the cusps was not seen or was mild, and the basal portion of the cusps was not thick; in the remaining two rats, the three cusps were totally thickened with moderate commissural fusion (Fig. 1). The tricuspid valve was slightly thick, usually when grade 2-3 P-valve lesions and/or VSD were present; the thickening occurred along the edges of

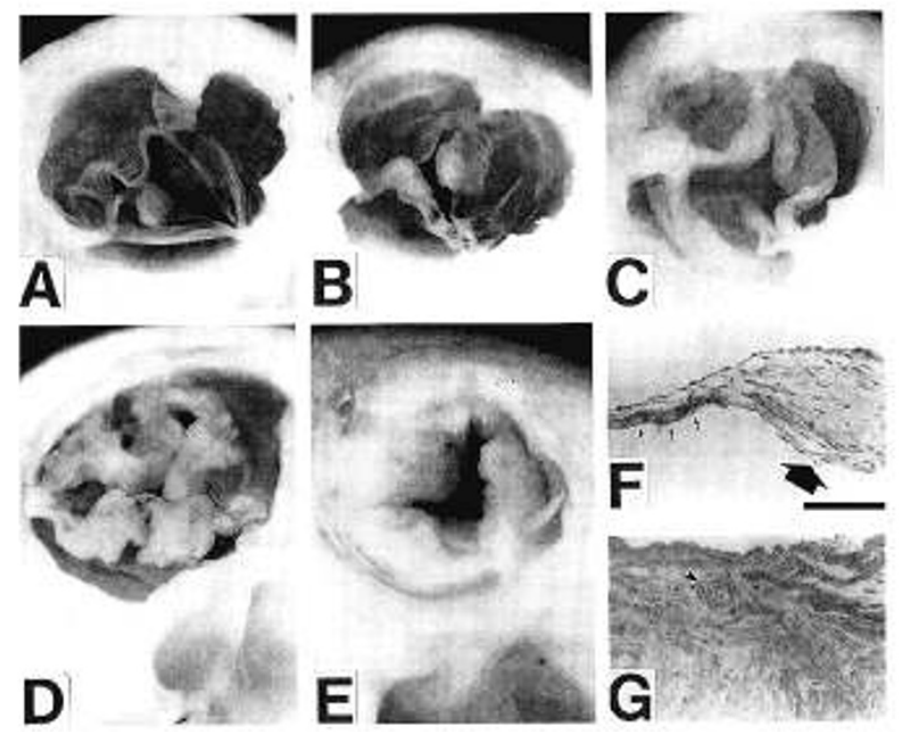

Fig. 1. Distal view of the P-valve. $A$, from a Wistar rat; there is no thickening except the nodule of the cusp. $B$, grade 1 thickening in WKY rats; thickening is restricted to the tip of the cusps. $C$, grade 2 thickening; the edges of the cusps are totally thickened with no commissural fusion. $D$, grade 3 thickening; all three cusps are severely thickened, and show cauliflower-like deformation with their apposition impaired but with no commissural fusion; note that the basal portion of the cusps is not thickened. $E$, grade 3 thickening; the three cusps are all very thick with their basal commissures fused. The aortic valve is not thick in $D$ and $E$. $F$, Histology of the P-valve of a Wistar rat. Masson-Goldner's trichrome staining. Scale, $100 \mu \mathrm{m}$ for $F$ and $G$. Arrow, nodule of the cusp. Small arrows, the outer half of the cusp membrane consists of dense collagen fibers (lamina fibrosa). $G$, from the same P-valve as in $E$. Dense and disarranged collagen fibers were distributed throughout the cusps. There are blood vessels (arrow head) with no infiltration of inflammatory cells. The endothelium is not clear.
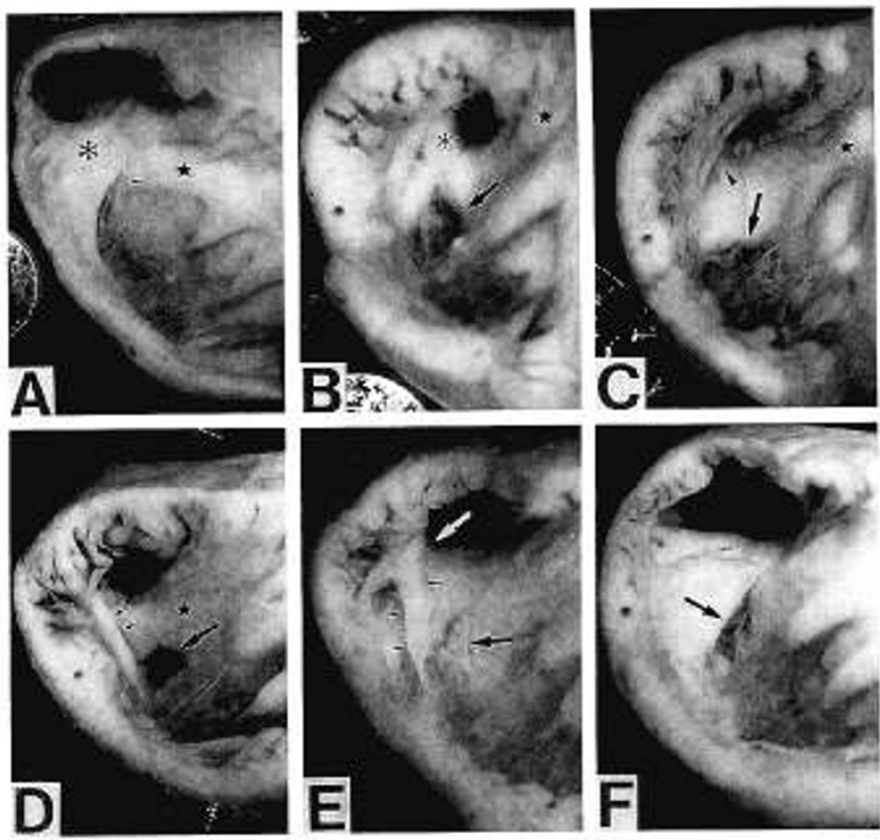

Fig. 2. View of the right side of the heart showing the basal half with the RVFW dissected. A, from a normal Wistar rat. Asterisk, crista supraventricularis. Star, septal band or trabecula septomarginalis. Arrow, papillary muscle of the conus. $B$, from a WKY rat. Arrow, VSD. There are membranous tissues in the VSD, which were continuous with the posterior cusp of the aorta, aortic ring, tricuspid valve, and the VSD edge. The POT is very narrow, being surrounded by anteriorly deviated septal (asterisk) and parietal bands (star), and thick RVFW with marked trabecular formation. The POT ostium is lined by fibrous nodules. The anterior papillary muscle is large. The papillary muscle of the conus is absent. $C$, from a WKY rat. There is a VSD with membranous tissues (arrow). Papillary muscles are large; chordae tendineae are well developed; and the edges of the tricuspid leaflets are mildly thickened. The POT is narrow, surrounded by the hypertrophied crista supraventricularis and circumferentially oriented trabeculae with a lining of fibrous nodules. The parietal (arrow head) and septal (star) bands appear to be anteriorly deviated, but do not constitute the POT ostium. $D$, There is a large VSD (arrow). The POT is narrow. The RVFW is thick due to the development of longitudinal trabeculae. The papillary muscle of the conus is absent; instead, there is a large papillary muscle originating from the conal RVFW, participating in narrowing of the POT instead of the parietal band. The septal band (star) is not anteriorly deviated. $E$, VSD is obstructed by dense membranous tissues (large arrow). POT narrowing is not severe, although the parietal band (white arrow) show slight anterior deviation, the RVFW is thick, and a large papillary muscle of the RVFW (small arrows) is present. $F$, VSD was closed by the posterior cusp of the aortic valve, which adhered to the membranous septum; the anterior tricuspid leaflet was continuous with them through a large chordal structure. The conal RVFW is slightly thick, but POT narrowing is not obvious.

the leaflets and at the points of attachment of the chordae tendineae (Fig. 2). Histologic study of the thickened valves showed dense and disarranged collagen fibers with increased vasculature, but no inflammatory cells (Fig. $1 F$ ). The aortic and/ or mitral valves were mildly thickened in several rats with VSD.

$V S D$. VSD of various sizes was present in $10 \%$ of the nonselected 198 rats (Table 1). In the entire series of 225 rats, all the VSD were accompanied by grade 1-3 P-valve thickening; the higher the grade, the more frequent was the association (Table 2 ). The right-sided orifice of the VSD was always located between the crista supraventricularis and the tricuspid ring (Fig. $2 B-D$ ), and the left-side orifice was immediately beneath the aortic valve, with the lumen most visible from the sinus of Valsalva when the posterior and right cusps were dissected (Fig. $3 B$ ). Longitudinal sectioning of the heart clearly indicated that the luminal axis was 
Table 1. Incidence of various abnormalities in 198 nonselected WKY rats*

\begin{tabular}{|c|c|c|c|}
\hline & & $\begin{array}{c}\text { WKY } \\
(n=198)\end{array}$ & $\begin{array}{c}\text { Wistar } \\
(n=30) \\
\end{array}$ \\
\hline \multicolumn{4}{|l|}{ P-valve thickening } \\
\hline grade 1 & & $(33 \%)$ & $(0 \%)$ \\
\hline grade 2 & & $(14 \%)$ & $(0 \%)$ \\
\hline grade 3 & & $(12 \%)$ & $(0 \%)$ \\
\hline VSD & {$[1-50] \dagger$} & $(10 \%)$ & $(0 \%)$ \\
\hline $\operatorname{POT}\left(\times 10^{3} \mu \mathrm{m}^{2}\right)$ & $100 \pm 39$ & $(59 \%)$ & $185 \pm 35$ \\
\hline $\begin{array}{l}\text { Absence of papillary } \\
\text { muscle of conus }\end{array}$ & & $(33 \%)$ & $(0 \%)$ \\
\hline $\begin{array}{l}\text { Papillary muscle of } \\
\text { RVFW }\end{array}$ & & $(62 \%)$ & $(0 \%)$ \\
\hline Heart wt (mg) & $8.09 \pm 1.42$ & $(9 \%)$ & $8.26 \pm 0.62$ \\
\hline IVS/LVFW & $1.12 \pm 0.11$ & $(53 \%)$ & $0.95 \pm 0.06$ \\
\hline $\begin{array}{l}\text { Transverse area of } \\
\text { RVFW }\left(\mathrm{mm}^{2}\right)\end{array}$ & $0.41 \pm 0.10$ & $(40 \%)$ & $0.34 \pm 0.03$ \\
\hline
\end{tabular}

* Data in WKY rats were obtained from 20 litters. Values, mean \pm $\mathrm{SD}$. The area values and heart wt were corrected by body wt $(\mathrm{g})$ raised to the power of $2 / 3$ and 0.9 , respectively.

$\dagger$ Brackets, range of lumen area $\left(\times 10^{3} \mu \mathrm{m}^{2}\right) ; 7$ VSD smaller than 1 were considered to be closed.

directed toward the aorta with the aortic cusps making the roof of the VSD (Fig. $3 D$ ). The IVS was deviated to the left due to the opening of the VSD to the sinus and was overriden by the aorta. Membranous tissues or small fibrous nodules were frequently plastered along the edge of the VSD, with the former usually being continuous with the tricuspid valve, membranous septum, aortic ring, and posterior and right aortic cusps (Figs. 2, $B$ and $C$; and $3 D$ ). Ventricular aneurysms (Fig. $2 E$ ) were present in 12 of the 198 rats. In addition, in 10 of the 198 rats, muscular defects were closed by the posterior and right aortic cusps, which adhered to the membranous septum and were continuous with the tricuspid valve through the chordae tendineae (Fig. $2 F$ ).

POT. The POT was abnormally narrow in $59 \%$ of the 198 WKY rats compared with the Wistar rats (Table 1). The narrowing was especially severe when VSD and/or grade $3 \mathrm{P}$-valve lesions were present; however, it was also present in more than half of the rats with neither of the two lesions (Table 2). In the hearts with POT narrowing, the crista supraventricularis was deviated more or less anteriorly (Fig. 2); sometimes the crista and well-developed parietal and septal bands surrounded the
POT, making the ostium very narrow (Fig. $2 B$ ), or the parietal band was inserted into the POT. Severe narrowing of POT was always accompanied by very thick conal RVFW with circumferential or longitudinal trabeculations (Fig. $2 C$ and $D$ ); the trabeculae sometimes traversed the middle of the POT ostium. In addition, small fibrous nodules frequently lined the perimeter of the narrow POT ostia (Fig. $2 B-D$ ). The infundibulum between the constricted POT ostia and the P-valves was not narrow and there was no thickening of the RVFW, producing an infundibular chamber (3). The size of the POT ostia showed significant negative correlations with the RVFW area $(-0.67<r<-0.50)$ and with the IVS/LVFW $(-0.45<r<-0.22)$.

Right ventricular papillary muscles. In the hearts with tricuspid valve thickening, the anterior and posterior papillary muscles were noticeably enlarged and the attached chordae were well developed (Fig. 2). The papillary muscle of the conus, which is always present in Wistar rats, was absent in $33 \%$ of the 20 litters of WKY rats (Fig. 2; Table 1). When this papillary muscle was absent, there were usually papillary muscles which originated from the RVFW or the large trabeculae in the POT, sometimes participating in the POT narrowing (Fig. $2 D$ and $E$ ). These two papillary muscles were both present in 71 rats, but both were absent in 9 of the total series of 225 WKY rats. In the latter cases, the anterior tricuspid leaflet was always continuous with the aortic ring and/or with the aortic cusps directly (Fig. $2 F$ ) or through the membranous tissue in the VSD lumen (Figs. $2 B$ and $C ; 3 D)$. Abnormality of the papillary muscles also was prevalently associated with VSD and severe P-valve lesions (Table 2).

Cardiac hypertrophy. Abnormally heavy hearts with DST (IVS/LVFW > 1.1 13 ) and RVH occurred in the 198 non-selected rats with a similar incidence and magnitude as in our previous study (13) (Table 1). These three abnormalities were more prevalent and marked when severe P-valve lesions and VSD were present (Table 2). However, in the total series of 225 rats, six of the 45 rats with abnormally heavy hearts and 67 of the 124 rats with DST did not have either VSD or grade 2-3 P-valve lesions (Table 2). In these six rats, ventricular aneurysms were present in two and muscular defects were closed by aortic cusps adherent to the membranous septum in one.

\section{DISCUSSION}

Various anomalous cardiac lesions, isolated or in association, occurred spontaneously in an inbred strain of rats. Because 30 of the 36 rats with VSD also had moderate to severe P-valve

Table 2. Relations between various abnormalities in 225 rats*

\begin{tabular}{|c|c|c|c|c|c|c|}
\hline $\begin{array}{c}\text { Lesion } \\
\text { Rats }(n)^{\prime \prime}\end{array}$ & [225] & $\begin{array}{c}\text { P-valve } \\
\text { grade } 0 \\
{[81]}\end{array}$ & $\begin{array}{c}\text { P-valve } \\
\text { grade } 1 \\
{[70]}\end{array}$ & $\begin{array}{c}\text { P-valve } \\
\text { grade } 2 \\
{[22]}\end{array}$ & $\begin{array}{c}\text { P-valve } \\
\text { grade } 3 \\
{[16]}\end{array}$ & $\begin{array}{l}\text { VSD } \\
{[36]}\end{array}$ \\
\hline VSD & {$[36]$} & (0) $0 \%$ & (6) $8 \dagger$ & (10) $31 \dagger \ddagger$ & (20) $56 \dagger$ & \\
\hline POT & [138] & $\begin{array}{l}(44) 54 \%^{\circ} \\
106 \pm 32\end{array}$ & $\begin{array}{l}\text { (38) } 54 \\
103 \pm 31\end{array}$ & $\begin{array}{l}\text { (12) } 55 \\
109 \pm 39\end{array}$ & $\begin{array}{l}\text { (11) } 69 \\
79 \pm 42\end{array}$ & $\begin{array}{l}\text { (33) } 92 \dagger \\
55 \pm 37 \dagger\end{array}$ \\
\hline Heart wt & [45] & $\begin{array}{l}\text { (1) } 1 \% \\
7.53 \pm 0.66\end{array}$ & $\begin{array}{l}\text { (5) } 7 \\
7.82 \pm 0.91\end{array}$ & $\begin{array}{l}\text { (2) } 9 \\
8.57 \pm 0.80 \dagger\end{array}$ & $\begin{array}{l}\text { (4) } 25 \dagger \\
8.75 \pm 0.87 \dagger\end{array}$ & $\begin{array}{l}\text { (33) } 92+t \\
12.1 \pm 2.2+\ddagger\end{array}$ \\
\hline IVS/LVFW & {$[124]$} & $\begin{array}{l}(34) 42 \% \\
1.09 \pm 0.09\end{array}$ & $\begin{array}{l}(33) 47 \\
1.09 \pm 0.09\end{array}$ & $\begin{array}{l}\text { (11) } 50 \\
1.14 \pm 0.06\end{array}$ & $\begin{array}{l}(11) 69 \\
1.16 \pm 0.14\end{array}$ & $\begin{array}{l}\text { (35) } 97 \dagger t \\
1.29 \pm 0.19+\ddagger\end{array}$ \\
\hline RVFW area & {$[105]$} & $\begin{array}{l}(23) 28 \% \\
0.37 \pm 0.04\end{array}$ & $\begin{array}{l}(23) 33 \\
0.38 \pm 0.04\end{array}$ & $\begin{array}{l}\text { (12) } 55 \dagger \\
0.44 \pm 0.07 \dagger\end{array}$ & $\begin{array}{l}\text { (11) } 69 \dagger \\
0.49 \pm 0.09 \dagger\end{array}$ & $\begin{array}{l}\text { (36) } 100 \dagger \ddagger \\
0.63 \pm 0.14 \uparrow \ddagger\end{array}$ \\
\hline $\begin{array}{l}\text { Absence of papillary muscle of } \\
\text { conus }\end{array}$ & {$[85]$} & (14) $17 \%$ & (17) 24 & (16) $73 \dagger \ddagger$ & (11) $69 \dagger$ & (27) $75 \dagger$ \\
\hline $\begin{array}{l}\text { Presence of papillary muscle of } \\
\text { RVFW }\end{array}$ & {$[151]$} & (44) $54 \%$ & (43) 61 & (18) $82 \dagger$ & (14) $88 \dagger$ & (32) $89 \dagger$ \\
\hline
\end{tabular}

* Brackets, number of rats with lesions or abnormal values. Parentheses, number of rats with both lesion on top and abnormality on left. $a$, number of rats with P-valve lesions do not include number of rats with VSD. Total of numbers in parentheses in a line equal number in bracket on left. $\%$, percentage of the number of rats in parentheses when rats have lesion on top: e.g. $b, 0 \%=[0 /(81+0)] \times 100 ; c, 54 \%=(44 / 81) \times 100$. \pm , mean \pm SD concerning item on left when rats have lesion on top.

$\dagger p<0.05$, for comparison with value in grade $0 \mathrm{P}$-valve lesion.

$+p<0.05$, for comparison with value in left column only. 

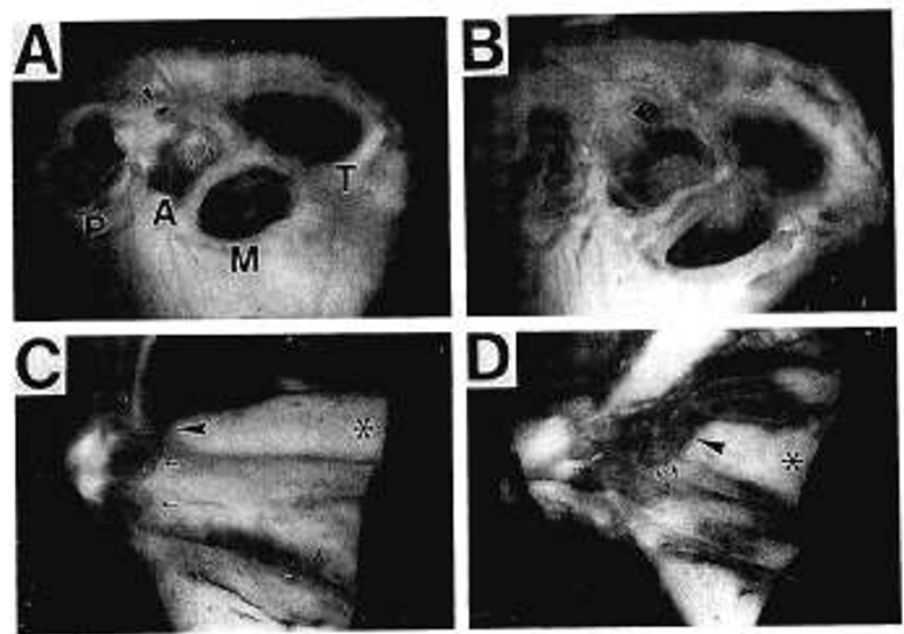

Fig. 3. $A$, from a normal Wistar rat, with the aortic valve dissected. $P, A, M$, and $T$; pulmonary, aortic, mitral, and tricuspid rings; arrows, left ventricular outflow tract; arrow head, right coronary artery. $B$, from a WKY rat. A VSD (arrow head) is visible through the sinus of Valsalva mainly beneath the dissected posterior aortic cusp. The IVS is deviated toward the left ventricle due to the presence of the VSD and is overridden by the aorta. The P-valve shows grade 2 thickening. $C$, longitudinal sectioning of a Wistar rat heart in a plane containing membranous septum and left ventricular outflow tract. Asterisk, IVS. Arrows, right and left cusps of the aortic valve. Arrow head, the IVS is continuous with the aortic ring through the membranous septum to which the tricuspid valve is attached. $D$, Longitudinal sectioning of a WKY heart in the same plane as in $C$. The continuity between the IVS and aortic ring is disturbed by the presence of a VSD (arrow head). The axis of the VSD is oriented toward the aorta, being roofed by the right and posterior cusps (dissected) of the aortic valve and by membranous tissue (arrows) between the cusps and the IVS. The tricuspid valve was continuous with the aortic ring, the aortic cusps, and the membranous tissue. There was no adhesion between the membranous tissue and aortic cusps.

thickening, POT narrowing, overriding of the aorta, and RVH, the 30 rats could be diagnosed as having TOF. The way in which the cardiac malformations are manifested in the rats seems similar to that in the Keeshond dogs (7-9). A wide spectrum of these lesions, except for narrowing of the POT, occurs within a single litter in the dog also, strongly suggesting that both animals are genetically predisposed to the development of the lesions. Familial studies in humans also have indicated that P-valve stenosis, VSD, and TOF occur significantly more frequently in families than in the general population $(14,15)$.

In our study, there was no typical dome-shaped P-valve stenosis that occurs often in man $(1,6)$; most of the P-valve lesions in the rats were similar to the type termed "pulmonary valvular dysplasia," which is characterized by three distinct cusps with thickening, but with no commissural fusion (16). The extensive studies on beagles and Keeshond dogs (7-10) demonstrated that both the dome-shaped stenosis and the valvular dysplasia occurred in dogs of the same litters, suggesting that the two types are not separate etiologic entities. The histologic appearance of the valves, with no inflammatory cells, was not consistent with bacterial or nonbacterial endocarditis (17). Neovascularization in dysplastic P-valves has been demonstrated in beagles (10) and in humans (16) also. It has also been noted that tricuspid valvular thickening is often associated with P-valve stenosis in man (1) and Keeshond dogs (8), possibly as a reaction to the excessive physical stresses placed on the valve apparatus (1).

The VSD in WKY rats correspond to membranous defects in Long-Evans rats (11), membranous (2) or perimembranous (4) defects in humans, and defects of the conotruncal septum in Keeshond dogs (7-9) depending on their location. The VSD always opened into the sinus of Valsalva through the cusps, with the axis of the lumen always oriented towards the sinus; this indicates that the size of the VSD conditions the degree of leftward deviation of the IVS and the overriding of the aorta. The growth of membranous tissue in the VSD, which may have been derived either from the tricuspid valve or from the remnants of the membranous septum (4), may be responsible for spontaneous closure of the defects $(4,7)$, together with the adhesion of the aortic cusps to the membranous septum.

POT narrowing is due partly to the anterior deviation of the crista supraventricularis with varying participation of the septal and parietal bands, and partly to the thickening of the outflow RVFW with marked trabecular formation. The POT were already more or less narrow in many of the WKY fetuses, particularly when VSD and/or P-valve lesions were present (Kuribayashi T, Shimoo K, Nakamura T, Hamaoka K, Nakagawa M, Ibata Y, Mizuta T, Komeda T, Nagaoka A, unpublished data). The drastic hemodynamic changes after birth will initiate a vicious circle; the narrowed POT, P-valve stenosis, and/or left to right shunting through the VSD will synergistically enhance the pressure overload on the right ventricle, which in turn may lead to further thickening of the outflow RVFW with various responses of the septal and parietal bands, promoting further constriction of the POT. The association of POT narrowing with P-valve stenosis (1) and hypertrophic cardiomyopathy (18) in humans has been attributed to RVH and DST, respectively.

The absence of papillary muscle of the conus is another feature in WKY rats as well as in Keeshond dogs (7-9). This occurred more frequently in the presence of P-valve lesions and VSD in both rats and dogs. A new finding in the rats was the presence of papillary muscles originating from the RVFW. The anterior tricuspid leaflet was continuous with either of the above two papillary muscles or aortic ring through the VSD lumen. It seems as though the anterior tricuspid leaflet requires some support for the anteriorly directed mechanical stress.

The abnormal wt of the heart, DST, and RVH in WKY rats became severe when VSD and/or P-valve stenosis was present, probably due to their hemodynamic effects. The prevalent association of DST and myocardial disarray with P-valve stenosis $(19-21)$, pulmonary hypertension $(18)$, and $\operatorname{TOF}(21,22)$ has been reported in humans also. It should be emphasized, however, that a considerable number of rats showed abnormally heavy hearts and DST in the absence of severe P-valve thickening and VSD. This is a situation not incompatible with hypertrophic cardiomyopathy, although other factors such as pulmonary hypertension remain to be excluded by hemodynamic studies.

The incidence of abnormally heavy hearts in the present 20 litters, as well as in the adult rats of 30 litters in the previous study $(8.3 \%)(2)$, was significantly lower than in the neonates 2 $4 \mathrm{~d}$ after birth (49.4\%) (Kuribayashi T, Shimoo K, Nakamura T, Hamaoka K, Nakagawa M, Ibata Y, Mizuta T, Komeda T, Nagaoka A, unpublished data). This lower incidence is not considered to be related to their mortality because the litter size in the 20 and 30 litters $\left(9.9 \pm 1.6,10 \pm 1.7^{13}\right)$ did not differ from that in the neonatal groups $(9.9 \pm 1.7)$, suggesting that much of the abnormal heaviness of the neonatal heart regresses with age. This regression seems to occur particularly when there is no VSD, and may be related to the postnatal closure of VSD. The higher incidence of cardiac hypertrophy in the neonates may be due to the fact that they were offspring of pairs selectively bred for hypertrophy. However, rats obtained from selective breeding over five generations with a litter size and incidence of VSD and P-valve lesions similar to those of the rats in this study did not have a higher incidence of hypertrophy when examined after 8 wk of age (Shimoo K, unpublished data); this ineffective selection may be reasonable considering that WKY/NCrj is an established inbred strain in which genes are presumed to be identical and present in homozygous form.

The WKY strain of rats is being used widely as a normotensive control in studies of spontaneously hypertensive rats (23). Pfeffer et al. (24) first described a subset of WKY rats with naturally occurring biventricular hypertrophy associated with left to right 
shunting, and they proposed it as an animal model of volumeload cardiac hypertrophy. Our study suggests that the shunting occurs through VSD, and it adds new possibilities for the WKY rat as an animal model of congenital cardiac malformations.

Acknowledgments. The authors wish to thank Dr. Alice Cary, Kyoto Baptist Hospital, and Dr. Masahiko Ando, Heart Institute of Japan, Tokyo Woman's Medical College, for reviewing the manuscript and Mr. Masayoshi Kitagawa, Laboratory Unit, Central Research Division, Takeda Chemical Industries, Ltd., for raising the rats.

\section{REFERENCES}

1. Emmanouillides GC, Baylen BG 1983 Pulmonary stenosis. In: Adams FH, Emmanouillides GC (eds) Heart Disease In Infants, Children, and Adolescents, 3rd ed. Williams \& Wilkins, Baltimore, pp 234-242

2. Graham TP, Bender HW, Spach MS 1983 Ventricular septal defect. In: Adams FH, Emmanouillides GC (eds) Heart Disease in Infants, Children, and Adolescents, 3rd ed. Williams \& Wilkins, Baltimore, pp 134-154

3. Guntheroth WG, Kawabori I, Baum D 1983 Tetralogy of Fallot. In: Adam FH, Emmanouillides GC (eds) Heart Disease in Infants, Children, and Adolescents, 3rd ed. Williams \& Wilkins, Baltimore, pp 215-228

4. Becker AE, Anderson RH 1981 Ventricular septal defect. In: Pathology of Congenital Heart Disease. Butterworth, London, pp 93-117

5. Becker AE, Anderson RH 1981 Abnormalities of the arterial valves. In: Pathology of Congenital Heart Disease. Butterworth, London, pp 179-186

6. Becker AE, Anderson RH 1981 Tetralogy of Fallot. In: Pathology of Congenital Heart Disease. Butterworth, London, pp 191-198

7. Patterson DF 1968 Epidemiologic and genetic studies of congenital heart disease in the dog. Circ Res 23:171-202

8. Patterson DF, Pyle RL, Van Mierop L, Melvin J, Olson M 1974 Hereditary defects of the conotruncal septum in Keeshond dogs: pathologic and genetic studies. Am J Cardiol 34:187-205

9. Van Mierop LHS, Patterson DF, Schnarr WR 1977 Hereditary conotruncal septal defects in Keeshond dogs: embryologic studies. Am J Cardiol 40:936950

10. Patterson DF, Haskins ME, Schnarr WR 1981 Hereditary dysplasia of the pulmonary valve in beagle dogs. Pathologic and genetic studies. Am J Cardiol 47:631-641

11. Fox MH 1967 Genetic transmission of congenital membranous ventricula septal defects in selectively inbred substrains of rats. Circ Res 20:422-433

12. Kuribayashi $T 1987$ Spontaneously occurring hypertrophic cardiomyopathy in the rat. I. Pathologic features. Jpn Circ J 51:573-588

13. Kuribayashi T, Mizuta T, Shimoo K, Kubota Y, Katsume H, Nakagawa M Ibata Y 1988 Spontaneously occurring hypertrophic cardiomyopathy in the rat. II. Distribution of, and correlations between, various cardiac abnormalities in the WKY/NCrj and its related strains. Jpn Circ J 52:1156-1170

14. Fraser FC, Hunter ADW 1975 Etiologic relations among categories of congenital heart malformations. Am J Cardiol 36:793-796

15. Nora JJ, Nora AH 1984 The genetic contribution to congenital heart disease. In: Nora JJ, Takao A (eds) Congenital Heart Disease. Causes and Processes. Furuta Publishing Co, New York, pp 3-13

16. Koretzky EK, Moller JH, Korns ME, Schwartz CJ, Edwards JE 1969 Congenital pulmonary stenosis resulting from dysplasia of valve. Circulation 40:4353

17. Oka M, Angrist A 1967 Mechanism of cardiac valvular fusion and stenosis Am Heart J 74:37-47

18. Roberts WC, Ferrans VJ 1975 Pathologic anatomy of the cardiomyopathies Idiopathic dilated and hypertrophic types, infiltrative types, and endomyocardial disease with and without eosinophilia. Hum Pathol 6:287-342

19. Maron BJ, Clark CE, Henry WL, Fukuda T, Edwards JE, Mathews Jr SC Redwood DR, Epstein SE 1977 Prevalence and characteristics of disproportionate ventricular septal thickening in patients with acquired or congenital heart diseases. Echocardiographic and morphologic findings. Circulation 55:489-496

20. Nora JJ, Lortscher RH, Spangler RD 1975 Echocardiographic studies of left ventricular disease in Ullrich-Noonan syndrome. Am J Dis Child 129:14171420

21. Larter WE, Allen HD, Sahn DJ, Goldberg SJ 1976 The asymmetrically hypertrophied septum. Further differentiation of its cause. Circulation 53:19-27

22. Kawai S, Okada R, Kitamura K, Suzuki A, Saito S 1984 A morphometrical study of myocardial disarray associated with right ventricular outflow tract obstruction. Jpn Circ J 48:445-456

23. Okamoto K, Aoki K 1963 Development of a strain of spontaneously hypertensive rats. Jpn Circ J 27:282-293

24. Pfeffer MA, Pfeffer JM, Dunn FG, Nishiyama K, Tsuchiya M, Frohlich ED 1979 Natural biventricular hypertrophy in normotensive rats. I. Physica and hemodynamic characteristics. Am J Physiol 236:H640-H643 